\title{
Ultrafast charge recombination in photoexcited Mott-Hubbard insulator
}

\author{
Zala Lenarčič ${ }^{1}$ and Peter Prelovšek ${ }^{1,2}$ \\ ${ }^{1} J$. Stefan Institute, SI-1000 Ljubljana, Slovenia and \\ ${ }^{2}$ Faculty of Mathematics and Physics, University of Ljubljana, SI-1000 Ljubljana, Slovenia
}

\begin{abstract}
We present a calculation of the recombination rate of the excited holon-doublon pairs based on the twodimensional model relevant for undoped cuprates which shows that fast processes, observed in pump-probe experiments on Mott-Hubbard insulators in picosecond range, can be explained even quantitatively with the multi-magnon emission. The precondition is the existence of the Mott-Hubbard bound exciton of the s-type. We find that its decay is exponentially dependent on the Mott-Hubbard gap and on the magnon energy, with a small prefactor which can be traced back to strong correlations and consequently large exciton-magnon coupling.
\end{abstract}

PACS numbers: 71.27.+a, 78.47.J-, 74.72.Cj

The advance in the ultrafast absorption spectroscopy allow for a direct access to excited states in a variety of materials as well as detailed studies of relaxation and thermalization processes. Correlated systems and Mott-Hubbard (MH) insulators are in this connection particularly of interest since here phenomena differ qualitatively from those well understood in band insulators and semiconductors. A prototype $\mathrm{MH}$ system are undoped cuprates as $\mathrm{YBa}_{2} \mathrm{Cu}_{3} \mathrm{O}_{6}, \mathrm{La}_{2} \mathrm{CuO}_{4}$ and $\mathrm{Nd}_{2} \mathrm{CuO}_{4}$ which have been investigated with femtosecond pump-probe spectroscopy [1-3] with an universal observation of ultrafast relaxation and recombination. The photoinduced carriers in undoped cuprates excited across the MH gap, which is in these systems of charge-transfer type, recombine in a picosecond range being a scale far below the usual physics of clean semiconductors with similar band gaps [4]. The origin of such ultrafast processes has been already qualitatively attributed to strong-correlation effects, in particular to larger energy of magnon excitations with the exchange energy $J$ and strong intrinsic coupling of charge carriers with spin fluctuations within the $\mathrm{MH}$ insulator [5], still no model-based theory was presented so far. Other $\mathrm{MH}$ insulators probed recently by ultrafast spectroscopy [6, 7] also exhibit fast-relaxation phenomena. Analogous are findings in the experiments with ultracold fermions in optical lattices where the decay of double occupancies [8, 9] are related to the correlated nature of the background state.

Due to strong Coulomb repulsion (strong correlations) excited quasiparticles within the $\mathrm{MH}$ insulators can be considered as empty sites (holons) and doubly occupied sites (doublons), respectively. In the following we consider as a prototype model the single-band Hubbard model on twodimensional (2D) square lattice in the regime of large $U \gg$ $t$, whereby the generalization to more appropriate chargetransfer model for cuprates is quite straightforward and the comparison with the parameters for actual materials can be also performed.

When interpreting the pump-probe experiments it is assumed that initially photoinduced mobile charges - holons and doublons - after very fast transient in the femtosecond range form a MH exciton, i.e., a bound holon-doublon (HD) pair. The existence and stability of the MH exciton has been previously addressed in connection with cuprates both in model studies [10-12] as well in the interpretation of optical response [7, 13] and of large Raman shift [14] with some conflicting conclusions. As a result of our study the disagreement can be reconciled with the observation that the ground state (g.s.) of bound HD pair is of the s-type, thus not observable in an optical absorption. Just such a s-type pair state appears to be the precondition for a fast non radiative recombination process. The evidence for the intermediate HD bound state can be pump-intensity independent recombination rates, apparently observed in experiments [3]. Nevertheless, the formation of an $\mathrm{MH}$ exciton is a nontrivial consequence of strong correlations and large charge-magnon coupling in 2D antiferromagnetic (AFM) background. The recombination of an $\mathrm{MH}$ exciton, i.e., a bound pair of a doublon in the upper Hubbard band (UHB) and a holon in a lower Hubbard band (LHB), across the MH gap $\Delta$ via the the multi-magnon emission is schematically presented in Fig. 1 Using the golden-rule ap-

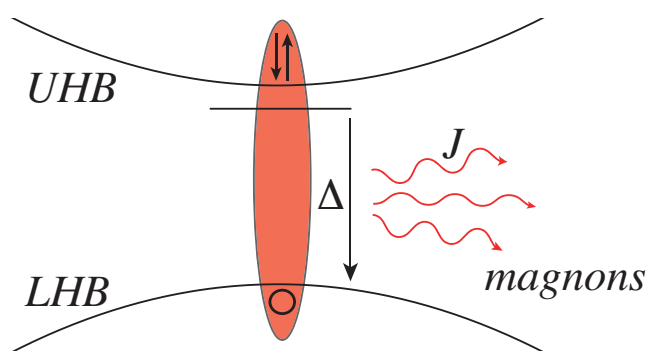

Figure 1: (Color online) The process of the Mott-Hubbard exciton recombination via the multi-magnon emission.

proach to the recombination rate $\Gamma=1 / \tau$ of the exciton (numericallly evaluated on small systems) we show that it is very large in comparison with clean semiconductors. In particular our results are consistent with the exponential dependence,

$$
\Gamma \propto \exp (-\alpha \Delta / J)
$$

so that enhanced $\Gamma$ has origin in large exchange scale $J$, but even more in small $\alpha \sim 0.5$ which can be again traced back to strong correlations. In spite of large $\Delta$ recombination times $\tau$ are in the picosecond range, consistent with pump-probe experiments in undoped cuprates [2, 3]. 
As the starting model for the study of photoexcited states in the $\mathrm{MH}$ insulator we use the single-band Hubbard model,

$$
H=-t \sum_{\langle i j\rangle s} c_{j s}^{\dagger} c_{i s}+U \sum_{i} n_{i \uparrow} n_{i \downarrow}
$$

where sums $\langle i j\rangle$ run over nearest-neighbor (n.n.) pairs of sites on a square 2D lattice. More realistic model for cuprates beyond the Hubbard model we comment furtheron. To reduce the complexity and as well to get correlation effects more transparent in the regime $U \gg t$ relevant for the $\mathrm{MH}$ insulator we perform the standard canonical transformation [15]. Within the lowest order in $t^{2} / U$ and neglecting the 3site terms one gets a generalized $t-J$ model for an insulator, including besides a spin-exchange term also both holon and doublon operators,

$$
\begin{aligned}
H_{t J}= & t \sum_{\langle i j\rangle s}\left(h_{j s}^{\dagger} h_{i s}-d_{j s}^{\dagger} d_{i s}+\text { h.c. }\right)+U \sum_{i} n_{d i}+ \\
& +J \sum_{\langle i j\rangle}\left(\mathbf{S}_{j} \cdot \mathbf{S}_{i}-\frac{1}{4} \delta_{1, n_{j} n_{i}}\right)
\end{aligned}
$$

where $h_{i s}^{\dagger}=c_{i s}\left(1-n_{i \bar{s}}\right)$ and $d_{i s}^{\dagger}=c_{i \bar{s}}^{\dagger} n_{i s}$ are projected fermionic operators, excluding double and unoccupied sites, respectively. Note that $h_{i s}^{\dagger}, d_{i s}^{\dagger}$ up to a sign represent creation holon and doublon operators on a site previously occupied by spin $s$ while $n_{d i}=\sum_{s} d_{i s}^{\dagger} d_{i s}$ and $n_{h i}=\sum_{s} h_{i s}^{\dagger} h_{i s}$ are the local number of doublons and holons, respectively. It is evident that $H_{t J}$ conserves the number of holons $N_{h}$ and doublons $N_{d}$. For the study of the HD recombination it is essential to include the 3-site term, which emerges after the canonical transformation [15] also within the order $t^{2} / U$. It has been so far mostly derived and considered for the hole-doped reference insulator [16, 17] and can be for the HD case (neglecting terms which do not represent HD recombination) written in a spin-invariant form,

$$
H_{3}=t_{3} \sum_{(i j k) s s^{\prime}}\left[h_{i s} d_{k s^{\prime}} \vec{\sigma}_{s \bar{s}^{\prime}} \cdot \mathbf{S}_{j}+\text { h.c. }\right],
$$

where $i \neq k$ are n.n. sites to $j$ and $\vec{\sigma}$ the Pauli matrix. Within the lowest order of the $t / U$ expansion one gets $t_{3}=$ $2 t^{2} / U=J / 2$. Evidently, $H_{3}$ creates and annihilates HD pairs which are on next-nearest-neighbor (n.n.n.) sites. It should be stressed that within a canonically transformed Hamiltonian there is no recombination term being on n.n. sites or being linear in $t$. We use furtheron the hopping as the unit of energy, $t=1$.

We proceed to the evaluation of the HD recombination process in two steps. To describe the relaxation of the photoexcited insulator through the intermediate bottleneck stage of an $\mathrm{MH}$ exciton we first establish the stability of the bound HD pair within the $H_{t J}$, Eq. (3), neglecting $H_{3}$. The problem is analogous to extensively studied case of the binding of two holes $N_{h}=2$ within the $t-J$ model [18], with an important distinction that holon and doublon are distinguishable particles and can form also $s$-type ( $\mathrm{A}_{1}$ symmetry) bound pair. In the $N_{h}=2$ problem the g.s. has the $d$-type bound state above the threshold $J>J_{c} \sim 0.3$ [18].

We study eigenstates of a single HD pair, i.e., $N_{h}=N_{d}=$ 1 by performing the exact diagonalization of $H_{t J}$ on small square and rectangular lattices with periodic boundary conditions using the Lanczos technique on $N \leq 26$ sites. As a criterion for binding we use the pair binding energy $\epsilon_{b}=$ $E_{0}^{h d}-E_{0}^{h}-E_{0}^{d}+E_{0}^{0}$ where $E_{0}^{h d}, E_{0}^{h}, E_{0}^{d}, E_{0}^{0}$ refer to the HD pair, single hole, single doublon and undoped AFM g. s., respectively. Within $H_{t J}$, Eq. (3), we also take into account $E_{0}^{d}=E_{0}^{h}+U$, while for $\epsilon_{0}^{h}=E_{0}^{h}-E_{0}^{0}$ we use known accurate fits [19]. Results for $\epsilon_{b}(J)$ obtained for $N=18,20,26$ are presented in Fig.2 for the lowest s-type state. Values for $\epsilon_{b}$ appear to be quite size independent and confirm previous findings [12] of the stability $\epsilon_{b}<0$ of the MH exciton. The state is, however, of an even symmetry and not observable in an optical transition from the insulator AFM state. On the other hand, the p-type state relevant for optical absorption reveals $\epsilon_{b} \gtrsim 0$, hence does not appear to be a bound one.

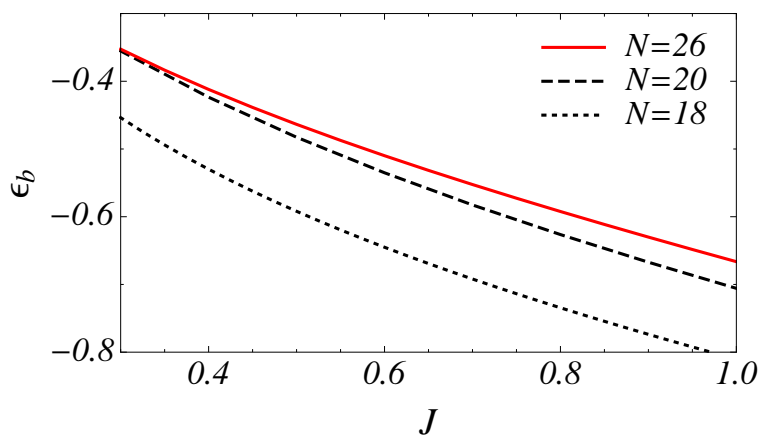

Figure 2: (Color online) Holon-doublon pair binding energy $\epsilon_{b}$ vs. $J$. Plotted are results for the s-type g.s. for $N=18,20,26$ lattices.

Another test for the HD binding are g.s. density correlations $D_{j}=\left\langle\psi_{0}^{h d}\left|n_{h j} n_{d 0}\right| \psi_{0}^{h d}\right\rangle$ (the position of the doublon chosen as the origin). In Fig. $3 D_{j}$ obtained (and symmetrized to recover the reflection symmetry) on $N=26$ for $J=0.4$ are consistent with the binding where the largest probability is for the HD pair being on a distance $d_{0}=\sqrt{2}$. On contrary, the unbound states (as the p-type lowest state) are characterized by largest $d_{0} \sim \sqrt{N}$ ) for a given lattice.

The next stage is to evaluate the recombination rate $\Gamma=$ $\tau^{-1}$ of the MH exciton using the Fermi golden rule with $H_{3}$, Eq. (4), serving as the perturbation,

$$
\Gamma=2 \pi \sum_{m}\left|\left\langle\psi_{m}^{0}\left|H_{3}\right| \psi_{0}^{h d}\right\rangle\right|^{2} \delta\left(E_{m}^{0}-E_{0}^{h d}\right),
$$

and transitions are into magnon states $\left|\psi_{m}^{0}\right\rangle$ within the undoped AFM. We note that Eq. (5) can be represented as a resolvent $\Gamma=-2 \operatorname{Im} C\left(\omega=E_{0}^{h d}-E_{0}^{0}\right)$,

$$
C(\omega)=\left\langle\psi_{0}^{h d}\left|H_{3} \frac{1}{\omega^{+}+E_{0}^{0}-H_{J}} H_{3}\right| \psi_{0}^{h d}\right\rangle,
$$




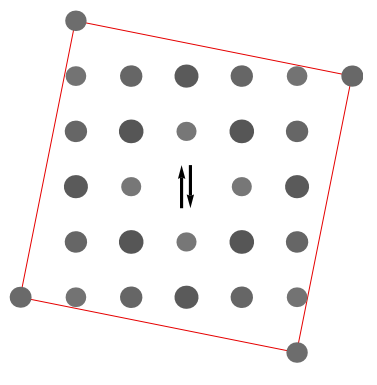

Figure 3: (Color online) Density correlations $D_{j}$ of the hole with respect to the doublon position for $J=0.4$, as calculated for $N=$ 26 lattice.

with $\omega^{+}=\omega+i \delta$. In the calculation only the AFM part $H_{J}$ of the $H_{t J}$, Eq. (3), is relevant. Eq. (6) is convenient for the evaluation within the Lanczos procedure, as usual for g.s. correlation functions [18, 20]. Starting with the perturbed $\left|\phi_{0}\right\rangle=H_{3}\left|\psi_{0}^{h d}\right\rangle$ the Lanczos procedure generates a tridiagonal matrix and $C(\omega)$ can be expressed in a continued-fraction form. While $\delta$ should be infinitesimally small thermodynamic limit $N \rightarrow \infty$ can be efficiently simulated by adopting finite $\delta \ll J$. Moreover, to avoid the influence of such a Lorentzian smoothing (note that final $\Gamma \ll 1$ ) we employ rather a Gaussian smoothing of poles produced after a finite number of Lanczos steps, $M \sim 150$.

The convergence of results with the system size is presented in Fig. 4 4 where $\Gamma$ is evaluated for fixed $J=0.4$ and different lattices with $N=20,24,26(N=24=4 \times 6$ sites is a rectangular lattice) sites. Results are shown versus the effective MH gap $\Delta=E_{0}^{h d}-E_{0}^{0}$ between the bound HD-pair state and the AFM g.s. (see Fig. 11). Taking $\Delta$ as a variable corresponds to assume $U$ in Eq. (3) as an independent parameter which could deviate from the relation $U=4 t^{2} / J$ obtained within the $U \gg t$ limit. We note from Fig. (4 that even small $\delta=0.07$ is enough to obtain continuous $\Gamma(\Delta)$ for the largest system $N=26$. Clearly, the system size $N$ should not be too small in order to accommodate enough magnons and to get reliable results in the relevant tails $\Gamma \ll 1$.

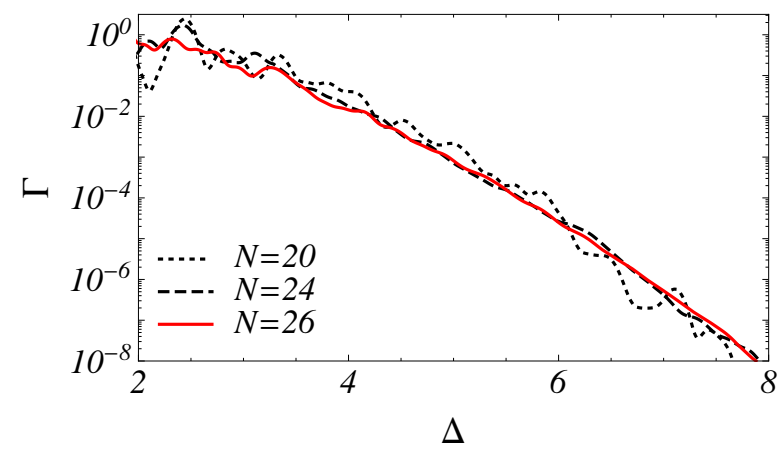

Figure 4: (Color online) Exciton recombination rate $\Gamma$ vs. gap $\Delta$ for $J=0.4$ and different planar systems with $N=20,24,26$ sites. Results are smoothed with $\delta=0.07$.
As the central result we present in Fig. $5 \mathrm{a}, \mathrm{b}$ calculated $\Gamma$ vs. $\Delta$ obtained on the largest system $N=26$ and different $J=0.3,0.4,0.6$. The dependence on $\Delta / J$ in the relevant regime $\Gamma(\Delta \sim U)$ is close to the exponential, Eq. (1), as presented in Fig. 56. Effective $\alpha$ in this case is within the window $0.3<\alpha<0.7$. Even better fit can be reached by following the perturbation-theory estimate for the probability of the generation of $n_{0}$ bosons $[8,9,21]$ where in our case $n_{0}=\Delta / J$,

$\Gamma \propto\left[\frac{1}{n_{0} !}\left(\frac{t}{J}\right)^{n_{0}}\right]^{2} \propto\left(\frac{e t}{n_{0} J}\right)^{2 n_{0}} \propto \exp \left[-\alpha_{0} \frac{\Delta}{J} \ln \frac{\Delta}{e t}\right]$.

Results presented in Fig. $5 \mathrm{~b}$ for $\Gamma$ vs. $(\Delta / J) \ln (\Delta /$ te $)$ reveal even better agreement, with quite universal $\alpha_{0} \sim 0.8$ (instead of $\alpha_{0}=2$ following from straight derivation of Eq. (7) ).
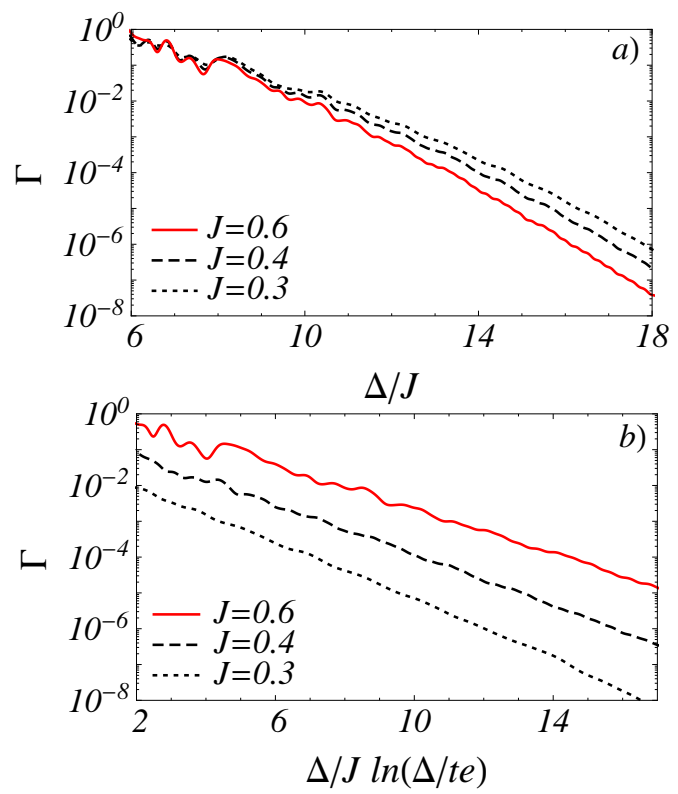

Figure 5: (Color online) Exciton recombination rate $\Gamma$ vs. a) $\Delta / J$ and b) $\Delta / J \ln (\Delta /$ te $)$ for different $J=0.3,0.4,0.6$ as calculated for $N=26$ sites.

Exponential dependence of $\Gamma$ on $J$, Eq. (1), is plausible since the recombination involves the multi-magnon emission. Taken at the face value within the reduced model, Eq. (3), with $\Delta=U-2\left|\epsilon_{0}^{h}\right|-\left|\epsilon_{b}\right|$ and $U=4 t^{2} / J$ the variation of $\Gamma$ with $J$ is even enhanced. Nevertheless, even for quite realistic value $J=0.4$ we get in this simplified case $\Delta / J \sim$ 17 and $\Gamma \sim 10^{-6}$ which is not unreasonably small taking into account large number of involved magnons. On the other hand, any decrease of the 'effective' gap $\Delta$ leads to a large enhancement of the rate $\Gamma$.

Before the application of our theory and results to actual MH-insulator materials and experiments we offer some explanation of substantial $\Gamma$ even for cases where evidently a large number of emitted magnons $n_{0} \sim \Delta / J \gg 1$ is required for the HD-pair recombination. It is already evident from numerous numerical [18] and analytical [22] studies of the single 
hole $N_{h}=1$ within the $t-J$ model that a holon (or doublon) perturbs strongly the AFM background in the strong correlation regime $J<t$. Even more this is the case for two (bound) holes $N_{h}=2$ [18] and for the present example of a bound HD pair, $N_{h}=N_{d}=1$. In Fig. 6 we present results for exchangeenergy deviations relative to the reference AFM g.s. Results are for $J=0.4$ and for the most probable HD configuration with the pair distance $d_{0}=\sqrt{2}$. It is quite evident that the recombination of a $\mathrm{HD}$ pair via $H_{3}$ (which just requires the holon and doublon being on n.n.n. sites) already leads to the generation of a large number of magnons $n_{0} \gg 1$.

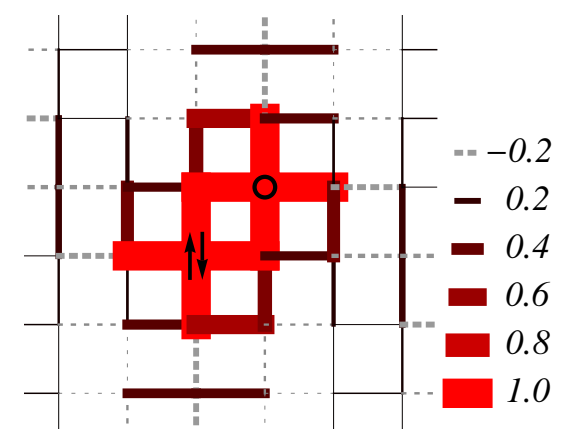

Figure 6: (Color online) Bond perturbations within the holondoublon pair g.s., presented for the most probable pair configuration. Results are for $J=0.4$ and deviations of bond energies relative to AFM g.s. are presented.

Related but not equivalent argument for the appreciable $\Gamma$ for cases $\Delta \gg J$ is small parameter $\alpha_{0}<1$ in the Ansatz (7) and even more $\alpha \sim 0.5$ (within the relevant regime of $J / t$ ) in Eq. (1). This can be considered as another manifestation of the strong coupling between the charge and spin excitations within the $\mathrm{MH}$ insulator as well as in strongly correlated systems in general.

Application to cuprates: Presented analysis and results can be generalized in quite straightforward way to insulators, where the $\mathrm{MH}$ gap represents charge-transfer excitations. In particular this includes insulating and superconducting cuprates, where more complete (three-band) model includes hybridized $\mathrm{Cu}$ d orbitals and $\mathrm{O}$ p orbitals. Although this goes beyond the prototype single-band Hubbard model in our study, through the Zhang-Rice mechanism [23] quasiparticles can be well described within the $t-J$ model both for doped holes and doped electrons [24]. Parameters are quite well established as $t \sim 0.35 \mathrm{eV}$ and $J / t \sim 0.4$ [24]. The three site term $\mathrm{H}_{3}$, Eq. (4), as well emerges from the threeband model [17], although $t_{3}$ can be quantitatively different but also less important entering $\Gamma$ in Eq. (5) only as a prefactor. So the essential generalization emerges from fact that the MH gap $\Delta$ and the 'effective bare' $U$ in the model, Eq. (3), are not directly related to $J$ and can be considered to large extent as independent parameters, taken from experiment or from more complete model. Optical MH gaps in undoped cuprates are experimentally known $\Delta_{0} \sim 1.5 \mathrm{eV}$, still differing between members of cuprate family. In order to get defined
$\Delta$ in Eq. (1) and in Fig. 1 we have to take into account that optical transitions are to HD p-type unbound states, so that $\Delta=\Delta_{0}-\left|\epsilon_{b}\right|$.

We first consider $\mathrm{Nd}_{2} \mathrm{CuO}_{4}$ with the optical gap $\Delta_{0}=$ $1.6 \mathrm{eV}$ [3] and $J=0.155 \mathrm{eV}$, so that we get $\Delta=4.1 t$ and $\Gamma \sim 2.2 \cdot 10^{-2}$ in units of $\tau_{0}^{-1}$ where $\tau_{0}=\hbar / t \sim 2$ fs. This yields final relaxation time $\tau=\Gamma^{-1} \sim 0.09 \mathrm{ps}$ which is surprisingly close to experimentally established $\tau \sim 0.2$ ps [3]. Analogous, for $\mathrm{La}_{2} \mathrm{CuO}_{4}$ with $\Delta_{0}=2 \mathrm{eV}$ and $J=0.133 \mathrm{eV}$ we get $\Delta=5.3 t$ and $\Gamma \sim 1.3 \cdot 10^{-4}$ which yields $\tau \sim 15$ ps. For the latter compound $\tau$ has not been well determined [3] but is considerably longer than in $\mathrm{Nd}_{2} \mathrm{CuO}_{4}$ consistent with our result.

Conclusions and discussion: The most important finding of this study is that multi-magnon emission can be quite an efficient mechanism for the nonradiative recombination of photoinduced charges in a $\mathrm{MH}$ insulator. This is in contrast to usual band insulators and semiconductors where the importance of analogous multi-phonon processes has not been established theoretically or experimentally [4], while such processes could be relevant for some novel structures as carbon nanotubes [21, 25]. The distinction of $\mathrm{MH}$ insulators is not just in larger boson scale $J$ compared to phonon energies but primarily in the strong coupling between charge quasiparticles (holes and doublons) and spin excitations. The main manifestation is in the established prefactor $\alpha_{0}<1$ in Eq. (7) as well as in $\alpha \sim 0.5$ in the exponential Eq. (1). It should be also reminded that in spite of strong correlations doped holes and doublons are quite mobile with modestly enhanced effective mass.

When interpreting recent pump-probe experiments on $\mathrm{MH}$ insulators, it should be reminded that we consider here only the bottleneck process of a $\mathrm{MH}$-exciton recombination. The initial relaxation of the pump-induced transient metallic-like collective state of holons and doublons [3] is expected to be much faster and has been already investigated although not fully settled in different model studies [26-30].

It should be noted that a similar mechanism of the HD-pair recombination has been recently considered as a candidate for the relaxation in fermionic cold-atom systems [8, 9] although it appears to be subdominant process to the kinetic-energy assisted decay which requires a finite density (metallic-like state) of nonequilibrium holons and doublons. The latter could emerge at effective $T>0$ or in systems far from equilibrium. On contrary, for 2D cuprates our study reveals that the multi-magnon mechanism of charge recombination and thermalization is very efficient and leads to ultrafast relaxation in the picosecond range as experimentally observed.

The authors acknowledge fruitful discussions with T. Tohyama. This work has been supported by the Program P10044 and the project J1-4244 of the Slovenian Research Agency (ARRS). 
[1] K. Matsuda et al., Phys. Rev. B 50, 4097 (1994).

[2] H. Okamoto et al., Phys. Rev. B 82, 060513 (2010)

[3] H. Okamoto et al., Phys. Rev. B 83, 125102 (2011)

[4] P. Yu and M. Cardona, Fundamentals of semiconductors: physics and materials properties (Springer, Berlin, 1996)

[5] M. Imada, A. Fujimori and Y. Tokura, Nature Phys. 7, 114 (2011).

[6] S. Wall, Nature Phys. 7, 114 (2011).

[7] F. Novelli et al., arXiv:1205.4609 (2012).

[8] N. Strohmaier et al., Phys. Rev. Lett. 104, 080401 (2010).

[9] R. Sensarma et al., Phys. Rev. B 82, 224302 (2010).

[10] P. Wróbel and R. Eder, Phys. Rev. B 66, 035111 (2002).

[11] T. Tohyama, H. Onodera, K. Tsutsui and S. Maekawa, Phys. Rev. Lett. 89, 257405 (2002).

[12] T. Tohyama, J. Phys. Soc. Jpn. 75, 34713 (2006).

[13] H. S. Choi et al., Phys. Rev. B 60, 4646 (1999).

[14] D. Salamon et al., Phys. Rev. B 51, 6617 (1995).

[15] K. A. Chao, J. Spałek and A. M. Oleś, J. Phys. C 10, L271 (1977).
[16] J. E. Hirsch, Phys. Rev. Lett. 54, 1317 (1985)

[17] A. Ramšak and P. Prelovšek, Phys. Rev. B 40, 2239 (1989).

[18] E. Dagotto, Rev. Mod. Phys. 66, 763-840 (1994).

[19] P. W. Leung and R. J. Gooding, Phys. Rev. B 52, R15711 (1995).

[20] P. Prelovšek and J. Bonča, arXiv:1111.5931 (2011).

[21] P. Avouris, M. Freitag and V. Perebeinos, Phys. Stat. Sol. 243, 3197 (2006).

[22] G. Martinez and P. Horsch, Phys. Rev. B 44, 317-331 (1991).

[23] F. C. Zhang and T. M. Rice, Phys. Rev. B 37, 3759 (1988).

[24] T. Tohyama, Phys. Rev. B 70, 174517 (2004).

[25] V. Perebeinos and P. Avouris, Phys. Rev. Lett. 101, 057401 (2008).

[26] A. Takahashi, H. Gomi and M. Aihara, Phys. Rev. Lett. 89, 206402 (2002).

[27] K. A. Al-Hassanieh, F. A. Reboredo, A. E. Feiguin, I. González and E. Dagotto, Phys. Rev. Lett. 100, 166403 (2008).

[28] Y. Kanamori, H. Matsueda and S. Ishihara, Phys. Rev. B 82, 115101 (2010).

[29] M. Eckstein and P. Werner, Phys. Rev. B 84, 035122 (2011).

[30] M. Eckstein and P. Werner, arXiv:1207.0402 (2012). 